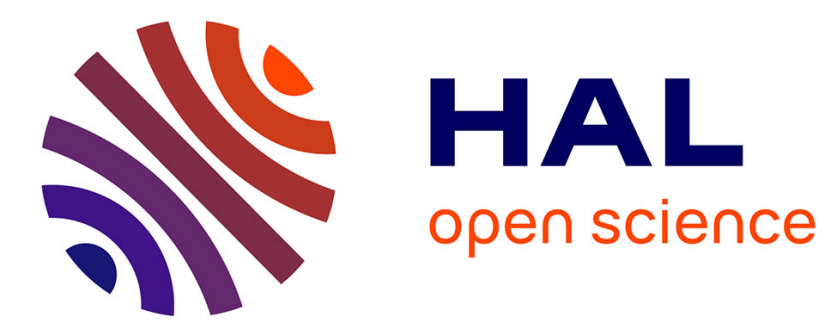

\title{
Third-order virtual measurements with signal injection
}

\author{
Dilshad Surroop, Pascal Combes, Philippe Martin, Pierre Rouchon
}

\section{To cite this version:}

Dilshad Surroop, Pascal Combes, Philippe Martin, Pierre Rouchon. Third-order virtual measurements with signal injection. CDC 2019 - 58th IEEE Conference on Decision and Control, Dec 2019, Nice, France. 10.1109/CDC40024.2019.9029751 . hal-02413822

\section{HAL Id: hal-02413822 \\ https://hal.science/hal-02413822}

Submitted on 16 Dec 2019

HAL is a multi-disciplinary open access archive for the deposit and dissemination of scientific research documents, whether they are published or not. The documents may come from teaching and research institutions in France or abroad, or from public or private research centers.
L'archive ouverte pluridisciplinaire HAL, est destinée au dépôt et à la diffusion de documents scientifiques de niveau recherche, publiés ou non, émanant des établissements d'enseignement et de recherche français ou étrangers, des laboratoires publics ou privés. 


\title{
Third-order virtual measurements with signal injection
}

\author{
Dilshad Surroop $^{1,2}$, Pascal Combes ${ }^{2}$, Philippe Martin ${ }^{1}$ and Pierre Rouchon ${ }^{1}$
}

\begin{abstract}
Signal injection was conceptualized in [1] as a method to make available an extra "virtual measurement", hence to simplify the design of a control law in particular when the system observability degenerates at a steady-state region of interest. In this paper, we show that the approach of [1] can be extended to produce yet others virtual measurements, thanks to an analysis based on third-order averaging.
\end{abstract}

\section{INTRODUCTION}

Signal injection is a control technique that has become widely used for the "sensorless" control of electrical motors at low velocity since its introduction by [2], [3] ("sensorless" meaning only the currents are measured, but neither the rotor position nor its velocity). Its consists in superimposing a fastvarying signal on the control, which creates some small ripple in the measured currents; this ripple contains information about the rotor position that can be used to suitably control the motor. At first sight, the method might seem peculiar to electrical motors, with a usually somewhat heuristic analysis. The essence of the method was then conceptualized in [1] as the creation of new "virtual measurements" which can be extracted from the actual measured output, hence providing means to overcome observability degeneracies; the main ingredient of the analysis is second-order averaging, following the ideas introduced in [4]. Developments following these ideas, with an application to magnetic levitation systems, can be found in [5], [6].

The purpose of this paper is to extend [1] by showing that more virtual measurements can be produced with a finer analysis of the ripple thanks to third-order averaging. To keep the computations as simple as possible and focus on the important ideas, we restrict to Single-Input Single-Output systems with a linear dynamics; nonlinear Multiple-Input Multiple-Output systems could nevertheless be addressed along the same lines. More precisely, consider the system

$$
\begin{aligned}
& \dot{x}=A x+B u \\
& y=h(x),
\end{aligned}
$$

where $(x, y, u)$ belongs to a compact subset of $\mathbb{R}^{n} \times \mathbb{R} \times \mathbb{R}$, and $A, B$ are constant matrices; the measured output $y=$ $h(x)$ is assumed smooth enough (e.g. at least $\mathcal{C}^{3}$ ). We show that by superimposing to the control $u$ a periodic signal with

\footnotetext{
1 D. Surroop, P. Martin and P. Rouchon are with the Centre Automatique et Systèmes, MINES ParisTech, PSL Research University, Paris, France \{dilshad.surroop, philippe.martin, pierre.rouchon \} @mines-paristech.fr

2 D. Surroop and P. Combes are with Schneider Toshiba Inverter Europe, Pacy-sur-Eure, France pascal.combes@schneider-electric.com
}

small period $\varepsilon$, we can make available the so-called virtual measurements

$$
\begin{aligned}
& Y_{1}=H_{1}(x):=\varepsilon h^{\prime}(x) B \\
& Y_{2}=H_{2}(x):=\frac{\varepsilon^{2}}{2}\left(h^{\prime \prime}(x) B\right) B \\
& Y_{3}=H_{3}(x):=\varepsilon^{2} h^{\prime}(x) A B,
\end{aligned}
$$

which can be used in addition to $Y_{0}=H_{0}(x):=h(x)$ to control (1a). The contribution with respect to [1] is threefold:

- an analysis of the output ripple by third-order averaging, with a simpler derivation (section II)

- a much more elaborated procedure to extract the virtual measurements from the output ripple (section III)

- a numerical simulation demonstrating the method is indeed effective, even though the output ripple may be very small and buried into noise (section IV).

\section{SignAl INJECTION AND THIRD-ORDER AVERAGING}

Assume we have designed a suitable control law

$$
\begin{aligned}
& u=\alpha(\eta, Y, t) \\
& \dot{\eta}=a(\eta, Y, t),
\end{aligned}
$$

where $\eta \in \mathbb{R}^{q}$ and $Y$ is the vector $\left(Y_{0}, Y_{1}, Y_{2}, Y_{3}\right)$. In other words, the closed-loop system

$$
\begin{aligned}
& \dot{\bar{x}}=A \bar{x}+B \alpha(\bar{\eta}, H(\bar{x}), t) \\
& \dot{\bar{\eta}}=a(\bar{\eta}, H(\bar{x}), t)
\end{aligned}
$$

has the desired exponentially stable behaviour, where $H:=$ $\left(H_{0}, H_{1}, H_{2}, H_{3}\right)$. We have changed the notation of the state to $(\bar{x}, \bar{\eta})$, so as to distinguish between the solutions of (3) and of (5) below.

Now consider the modified control law

$$
\begin{aligned}
u & =\alpha(\eta, \bar{H}(x), t)+s_{0}\left(\frac{t}{\varepsilon}\right) \\
\dot{\eta} & =a(\eta, \bar{H}(x), t) \\
\bar{H}(x) & =H\left(x-\varepsilon B s_{1}\left(\frac{t}{\varepsilon}\right)-\varepsilon^{2} A B s_{2}\left(\frac{t}{\varepsilon}\right)\right)+\mathcal{O}_{\infty}\left(\varepsilon^{3}\right),
\end{aligned}
$$

where $s_{0}$ is a 1-periodic function with zero mean, $s_{1}$ is the primitive of $s_{0}$ with zero mean, and $s_{2}$ the primitive of $s_{1}$ with zero mean (notice $s_{1}$ and $s_{2}$ are also 1-periodic); and $\mathcal{O}_{\infty}$ denotes the uniform "big O" symbol of analysis, namely $f(z, \varepsilon)=\mathcal{O}_{\infty}(\varepsilon)$ if there exists $K>0$ independant of $z$ and $\varepsilon$ such that $\|f(z, \varepsilon)\| \leq K \varepsilon$. The closed-loop system then reads

$$
\begin{aligned}
\dot{x} & =A x+B \alpha(\eta, \bar{H}(x), t)+B s_{0}\left(\frac{t}{\varepsilon}\right) \\
\dot{\eta} & =a(\eta, \bar{H}(x), t) \\
\bar{H}(x) & =H\left(x-\varepsilon B s_{1}\left(\frac{t}{\varepsilon}\right)-\varepsilon^{2} A B s_{2}\left(\frac{t}{\varepsilon}\right)\right)+\mathcal{O}_{\infty}\left(\varepsilon^{3}\right) .
\end{aligned}
$$


Theorem 1: Let $(x(t), \eta(t))$ and $(\bar{x}(t), \bar{\eta}(t))$ be respectively the solutions of (5) and (3), with initial condition $(x(0), \eta(0))$ and $(\bar{x}(0), \bar{\eta}(0))=\left(x(0)-\varepsilon B s_{1}(0)-\right.$ $\left.\varepsilon^{2} A B s_{2}(0), \eta(0)\right)$. Then for all $t \geq 0$,

$$
\begin{aligned}
x(t)= & \bar{x}(t)+\varepsilon B s_{1}\left(\frac{t}{\varepsilon}\right)+\varepsilon^{2} A B s_{2}\left(\frac{t}{\varepsilon}\right)+\mathcal{O}_{\infty}\left(\varepsilon^{3}\right) \\
\eta(t)= & \bar{\eta}(t)+\mathcal{O}_{\infty}\left(\varepsilon^{3}\right) \\
y(t)= & H_{0}(\bar{x}(t))+H_{1}(\bar{x}(t)) s_{1}\left(\frac{t}{\varepsilon}\right)+H_{2}(\bar{x}(t)) s_{1}^{2}\left(\frac{t}{\varepsilon}\right) \\
& +H_{3}(\bar{x}(t)) s_{2}\left(\frac{t}{\varepsilon}\right)+\mathcal{O}_{\infty}\left(\varepsilon^{3}\right) .
\end{aligned}
$$

Proof: The proof is an application of higher-order averaging for differential equations [7, section 2.9], with slow time dependance [7, section 3.3]: consider the two equations

$$
\begin{aligned}
& \frac{d \bar{X}}{d \sigma}(\sigma)=\varepsilon f^{1}(\bar{X}, \varepsilon \sigma, \sigma)+\mathcal{O}_{\infty}\left(\varepsilon^{4}\right) \\
& \frac{d \widetilde{X}}{d \sigma}(\sigma)=\varepsilon f^{1}(\widetilde{X}, \varepsilon \sigma, \sigma)+\varepsilon^{3} k^{3}(\widetilde{X}, \varepsilon \sigma, \sigma)+\mathcal{O}_{\infty}\left(\varepsilon^{4}\right),
\end{aligned}
$$

where $f^{1}, k^{3}$ are $T$-periodic with respect to their third variable and $k^{3}$ has zero-mean with respect to its third variable; according to [7, theorem 2.9.2], the solutions of these two equations starting from the same initial condition are related by

$$
\widetilde{X}(\sigma)=\bar{X}(\sigma)+\mathcal{O}_{\infty}\left(\varepsilon^{3}\right)
$$

on the timescale $1 / \varepsilon$. It is possible to extend this relation to an infinite timescale, provided that the averaged system has an exponentially stable equilibrium, and that the initial condition is in a compact subset of the region of attraction of this equilibrium, along the lines of [1, lemma 2].

In our case, we first rewrite (5) in the fast timescale $\sigma:=t / \varepsilon$

$$
\begin{aligned}
& \frac{d x}{d \sigma}=\varepsilon\left[A x+B \alpha(\eta, \bar{H}(x), \varepsilon \sigma)+B s_{0}(\sigma)\right] \\
& \frac{d \eta}{d \sigma}=\varepsilon a(\eta, \bar{H}(x), \varepsilon \sigma) .
\end{aligned}
$$

We introduce the new coordinates $(\widetilde{x}, \widetilde{\eta})$ such that

$$
\left(\begin{array}{l}
\widetilde{x} \\
\widetilde{\eta}
\end{array}\right)=\left(\begin{array}{l}
x \\
\eta
\end{array}\right)-\varepsilon\left(\begin{array}{c}
B s_{1}(\sigma) \\
0
\end{array}\right)-\varepsilon^{2}\left(\begin{array}{c}
A B s_{2}(\sigma) \\
0
\end{array}\right) .
$$

Notice (5c) simply reads

$$
\bar{H}(x)=H(\widetilde{x})+\mathcal{O}_{\infty}\left(\varepsilon^{3}\right) .
$$

Differentiating with respect to $\sigma$ and expanding to third order yields the system in the new coordinates

$$
\begin{aligned}
\dot{\widetilde{x}} & =\varepsilon[A \widetilde{x}+B \alpha(\widetilde{\eta}, H(\widetilde{x}), \varepsilon \sigma)] \\
& +\varepsilon^{3} A^{2} B s_{2}(\sigma)+\mathcal{O}_{\infty}\left(\varepsilon^{4}\right) \\
\dot{\widetilde{\eta}} & =\varepsilon a(\widetilde{\eta}, H(\widetilde{x}), \varepsilon \sigma)+\mathcal{O}_{\infty}\left(\varepsilon^{4}\right) .
\end{aligned}
$$

By (7), we then get

$$
\begin{aligned}
& \widetilde{x}=\bar{x}+\mathcal{O}_{\infty}\left(\varepsilon^{3}\right) \\
& \widetilde{\eta}=\bar{\eta}+\mathcal{O}_{\infty}\left(\varepsilon^{3}\right) .
\end{aligned}
$$

Replacing $(\widetilde{x}, \widetilde{\eta})$ by $(x, \eta)$ using transformation (9) in the two previous equations, we have the desired result

$$
\begin{aligned}
& x=\bar{x}+\varepsilon B s_{1}(\sigma)+\varepsilon^{2} A B s_{2}(\sigma)+\mathcal{O}_{\infty}\left(\varepsilon^{3}\right) \\
& \eta=\bar{\eta}+\mathcal{O}_{\infty}\left(\varepsilon^{3}\right) .
\end{aligned}
$$

Plugging this expression for $x$ in $y=h(x)$ and Taylor expanding to second order yields (6c).

The practical use of the theorem is the following. Assume we can compute a third-order estimate $\widehat{Y}$ of $Y$ from the knowledge of only the actual measurement $y$. Using the relation between $x$ and $\bar{x}$, we have

$$
\begin{aligned}
\widehat{Y} & =H(\bar{x})+\mathcal{O}_{\infty}\left(\varepsilon^{3}\right) \\
& =\bar{H}(x)+\mathcal{O}_{\infty}\left(\varepsilon^{3}\right)
\end{aligned}
$$

The control law with signal injection

$$
\begin{aligned}
& u=\alpha(\eta, \widehat{Y}, t)+s_{0}\left(\frac{t}{\varepsilon}\right) \\
& \dot{\eta}=a(\eta, \widehat{Y}, t)
\end{aligned}
$$

is then practically implementable and will control (1) as desired in average.

\section{Demodulation of the Virtual MEASUREMENTS}

In this section, we show how to estimate the virtual measurements from the ripple in the actual measurement. Consider the composite signal

$$
\begin{aligned}
y(t)= & Y_{0}(t)+Y_{1}(t) s_{1}\left(\frac{t}{\varepsilon}\right) \\
& +Y_{2}(t) s_{1}^{2}\left(\frac{t}{\varepsilon}\right)+Y_{3}(t) s_{2}\left(\frac{t}{\varepsilon}\right)+\mathcal{O}_{\infty}\left(\varepsilon^{3}\right),
\end{aligned}
$$

where $Y_{0}, \ldots, Y_{3}$ are in $\mathcal{C}^{3}$ and $Y_{0}^{(3)}, \ldots, Y_{3}^{(3)}$ are bounded. Theorem 2 below states that $Y_{0}, \ldots, Y_{3}$ can be estimated at third order thanks to periodic low-pass filters.

\section{A. Decomposition on an orthogonal basis}

We first rewrite $y$ as a decomposition on the periodic orthogonal signals $\left(1, s_{1}, s_{2}, S_{1}\right)$,

$$
\begin{aligned}
y(t)= & \widetilde{Y_{0}}(t)+\widetilde{Y_{1}}(t) s_{1}\left(\frac{t}{\varepsilon}\right) \\
& +\widetilde{Y_{2}}(t) S_{1}\left(\frac{t}{\varepsilon}\right)+\widetilde{Y_{3}}(t) s_{2}\left(\frac{t}{\varepsilon}\right)+\mathcal{O}_{\infty}\left(\varepsilon^{3}\right) .
\end{aligned}
$$

The signals $1, s_{1}, s_{2}$ are orthogonal (for the scalar product $\left.\langle f, g\rangle=\int_{0}^{1} f(\tau) g(\tau) d \tau\right)$; indeed, $\left\langle 1, s_{1}\right\rangle=\left\langle 1, s_{2}\right\rangle=0$ since $s_{1}, s_{2}$ have zero mean, and $\left\langle s_{1}, s_{2}\right\rangle=\frac{1}{2} \int_{0}^{1}\left(s_{1}^{2}\right)^{\prime}(\sigma) d \sigma=0$. $S_{1}$ is then obtained from $s_{1}^{2}$ by Gram-Schmidt orthogonalization,

$$
S_{1}(t):=s_{1}^{2}(t)-\overline{s_{1}^{2}}-\frac{\left\langle s_{1}^{2}, s_{1}\right\rangle}{\bar{s}_{1}^{2}} s_{1}(t)-\frac{\left\langle s_{1}^{2}, s_{2}\right\rangle}{s_{2}^{2}} s_{2}(t) .
$$

As a consequence, the "coordinates" $\tilde{Y}_{i}$ are

$$
\begin{aligned}
& \widetilde{Y_{0}}(t)=Y_{0}(t)+\overline{s_{1}^{2}} Y_{2}(t) \\
& \widetilde{Y_{1}}(t)=Y_{1}(t)+\frac{\left\langle s_{1}^{2}, s_{1}\right\rangle}{\overline{s_{1}^{2}}} Y_{2}(t) \\
& \widetilde{Y_{2}}(t)=Y_{2}(t) \\
& \widetilde{Y_{3}}(t)=Y_{3}(t)+\frac{\left\langle s_{1}^{2}, s_{2}\right\rangle}{\overline{s_{2}^{2}}} Y_{2}(t) .
\end{aligned}
$$




\section{B. Extraction of the $Y_{i}$ using iterated moving averages}

We now turn to the design of the demodulating filters, which are based on iterated moving averages of the form

$$
\begin{aligned}
M_{\varphi}^{1}(t) & :=\frac{1}{\varepsilon} \int_{t-\varepsilon}^{t} \varphi(\sigma) d \sigma \\
M_{\varphi}^{k}(t) & :=\frac{1}{\varepsilon} \int_{t-\varepsilon}^{t} M_{\varphi}^{k-1}(\sigma) d \sigma, \quad k>1 .
\end{aligned}
$$

We first recall a basic result on finite differences.

Lemma 1: Let $\varphi$ be $\mathcal{C}^{3}$ with $\varphi^{(3)}$ bounded. Then the $\mathrm{p}^{\text {th }}$ order backward difference

$$
\Delta_{\varphi}^{p}(t):=\sum_{i=0}^{p}\left(\begin{array}{l}
p \\
i
\end{array}\right)(-1)^{i} \varphi(t-i \varepsilon)
$$

satisfies

$$
\left\|\Delta_{\varphi^{(3-p)}}^{p}\right\|_{\infty} \lesssim \varepsilon^{p}\left\|\varphi^{(3)}\right\|_{\infty}, \quad p=0, \ldots, 3 ;
$$

$\varphi \lesssim \psi$ means there exists $K>0$ such that $\varphi \leq K \psi$.

Proof: For simplicity, we just prove as an example the case $p=2$. By the Taylor-Lagrange formula, there exists $t_{1} \in[t-\varepsilon, t]$ and $t_{2} \in[t-2 \varepsilon, t]$ such that,

$$
\begin{aligned}
\varphi^{\prime}(t-\varepsilon) & =\varphi^{\prime}(t)-\varepsilon \varphi^{\prime \prime}(t)+\frac{\varepsilon^{2}}{2} \varphi^{(3)}\left(t_{1}\right) \\
\varphi^{\prime}(t-2 \varepsilon) & =\varphi^{\prime}(t)-2 \varepsilon \varphi^{\prime \prime}(t)+2 \varepsilon^{2} \varphi^{(3)}\left(t_{2}\right)
\end{aligned}
$$

Therefore,

$$
\Delta_{\varphi^{\prime}}^{p}(t)=-\varepsilon^{2} \varphi^{(3)}\left(t_{1}\right)+2 \varepsilon^{2} \varphi^{(3)}\left(t_{2}\right)
$$

hence the desired inequality

$$
\left\|\Delta_{\varphi^{\prime}}^{p}\right\|_{\infty} \leq 3 \varepsilon^{2}\left\|\varphi^{(3)}\right\|_{\infty} .
$$

This lemma is used to prove the following result, which has an important role on the filter design.

Lemma 2: Let $\varphi$ be in $\mathcal{C}^{3}$ with $\varphi^{(3)}$ bounded, and $\zeta_{0}$ be a 1-periodic function with zero mean. Then,

$$
\left\|M_{\varphi \check{\varphi} \zeta_{0}}^{3}\right\|_{\infty} \lesssim \varepsilon^{3}\left\|\varphi^{(3)}\right\|_{\infty}\left\|\zeta_{3}\right\|_{\infty}
$$

with $\zeta_{j+1}$ the zero-mean primitive of $\zeta_{j}$, and $\check{\zeta}(t):=\zeta\left(\frac{t}{\varepsilon}\right)$.

Proof: Integrating by parts three times and using the periodicity of $\zeta_{j}$ gives

$$
\begin{aligned}
M_{\varphi \check{\zeta_{0}}}(t) & =\Delta_{\varphi}^{1}(t) \zeta_{1}\left(\frac{t}{\varepsilon}\right)-\varepsilon \Delta_{\varphi^{\prime}}^{1}(t) \zeta_{2}\left(\frac{t}{\varepsilon}\right) \\
& +\varepsilon^{2} \Delta_{\varphi^{\prime \prime}}^{1}(t) \zeta_{3}\left(\frac{t}{\varepsilon}\right)-\varepsilon^{2} \int_{t-\varepsilon}^{t} \varphi^{(3)}(\sigma) \zeta_{3}\left(\frac{\sigma}{\varepsilon}\right) d \sigma .
\end{aligned}
$$

We now dominate the last two terms: clearly,

$$
\left|\varepsilon^{2} \int_{t-\varepsilon}^{t} \varphi^{(3)}(\sigma) \zeta_{3}\left(\frac{\sigma}{\varepsilon}\right)\right| \leq \varepsilon^{3}\left\|\varphi^{(3)}\right\|_{\infty}\left\|\zeta_{3}\right\|_{\infty} ;
$$

lemma 1 applied to the third term yields

$$
\left|\varepsilon^{2} \Delta_{\varphi^{\prime \prime}}^{1}(t) \zeta_{3}\left(\frac{t}{\varepsilon}\right)\right| \lesssim \varepsilon^{3}\left\|\varphi^{(3)}\right\|_{\infty}\left\|\zeta_{3}\right\|_{\infty} .
$$

Notice that if $\|f\|_{\infty} \leq K$ then $\left\|M_{f}^{p}\right\|_{\infty} \leq K$; therefore applying $M^{1}$ and $M^{2}$ to these two terms yields the same bounds.
Consider next the second term of (13); its moving average is

$$
-\varepsilon M_{\Delta_{\varphi^{\prime}}^{1} \check{\zeta}_{2}}(t)=-\varepsilon \Delta_{\varphi^{\prime}}^{2} \zeta_{3}\left(\frac{t}{\varepsilon}\right)+\varepsilon \int_{t-\varepsilon}^{t} \Delta_{\varphi^{\prime \prime}}^{1}(\sigma) \zeta_{3}\left(\frac{\sigma}{\varepsilon}\right) d \sigma .
$$

Using again lemma 1, the two terms of the right-hand side are similarly dominated by $\left\|\varphi^{(3)}\right\|_{\infty}\left\|\zeta_{3}\right\|_{\infty}$, and so are their moving averages.

Finally, consider the first term of (13). Its moving average is

$$
\begin{aligned}
M_{\Delta_{\varphi}^{1} \check{\zeta_{1}}}(t)= & \Delta_{\varphi}^{2}(t) \zeta_{2}\left(\frac{t}{\varepsilon}\right)-\varepsilon \Delta_{\varphi^{\prime}}^{2}(t) \zeta_{3}\left(\frac{t}{\varepsilon}\right) \\
& +\varepsilon \int_{t-\varepsilon}^{t} \Delta_{\varphi^{\prime \prime}}^{1}(\sigma) \zeta_{3}\left(\frac{\sigma}{\varepsilon}\right) d \sigma
\end{aligned}
$$

Likewise, by lemma 1 , the last two terms of the right-hand side are also dominated by $\left\|\varphi^{(3)}\right\|_{\infty}\left\|\zeta_{3}\right\|_{\infty}$, and so are their moving average; as for the first term, integrating by parts its moving average yields

$$
M_{\Delta_{\varphi}^{2} \check{\zeta_{2}}}(t)=\Delta_{\varphi}^{3}(t) \zeta_{3}\left(\frac{t}{\varepsilon}\right)-\int_{t-\varepsilon}^{t} \Delta_{\varphi^{\prime}}^{2}(\sigma) \zeta_{3}\left(\frac{\sigma}{\varepsilon}\right) d \sigma .
$$

Using lemma 1 to the right-hand side ends the proof.

We then recover the $\widetilde{Y}_{i}$ from linear combination of shifted $M_{y}^{3}$ thanks to the following lemma.

Lemma 3: Let $\varphi$ be in $\mathcal{C}^{3}$ with $\varphi^{(3)}$ bounded. Define

$$
P_{\varphi}(t):=\frac{17}{4} M_{\varphi}^{3}(t)-5 M_{\varphi}^{3}(t-\varepsilon)+\frac{7}{4} M_{\varphi}^{3}(t-2 \varepsilon),
$$

Then the operator $\varphi \rightarrow P_{\varphi}$ is the identity up to third order,

$$
P_{\varphi}(t)=\varphi(t)+\mathcal{O}_{\infty}\left(\varepsilon^{3}\right) .
$$

Proof: Again, consider first a single moving average of $\varphi$. Doing a change of variable in the integral and computing the Taylor expansion of $\varphi$ gives

$$
\begin{aligned}
\frac{1}{\varepsilon} \int_{t-\varepsilon}^{t} & \varphi(\sigma) d \sigma=\frac{1}{\varepsilon} \int_{0}^{\varepsilon} \varphi(t-\sigma) d \sigma \\
& =\frac{1}{\varepsilon} \int_{0}^{\varepsilon}\left[\varphi(t)-\sigma \varphi^{\prime}(t)+\frac{\sigma^{2}}{2} \varphi^{\prime \prime}(t)\right] d \sigma+\mathcal{O}_{\infty}\left(\varepsilon^{3}\right) \\
& =\varphi(t)-\frac{\varepsilon}{2} \varphi^{\prime}(t)+\frac{\varepsilon^{2}}{6} \varphi^{\prime \prime}(t)+\mathcal{O}_{\infty}\left(\varepsilon^{3}\right) .
\end{aligned}
$$

We iterate the previous calculations to get the expressions of $M_{\varphi}^{2}$ and $M_{\varphi}^{3}$,

$$
\begin{aligned}
M_{\varphi}^{2}(t)= & M_{\varphi}(t)-\frac{\varepsilon}{2} M_{\varphi^{\prime}}(t)+\frac{\varepsilon^{2}}{6} M_{\varphi^{\prime \prime}}(t)+\mathcal{O}_{\infty}\left(\varepsilon^{3}\right) \\
& +\varphi(t)-\varepsilon \varphi^{\prime}(t)+\frac{7}{12} \varphi^{\prime \prime}(t)+\mathcal{O}_{\infty}\left(\varepsilon^{3}\right) \\
M_{\varphi}^{3}(t)= & \varphi(t)-\frac{3}{2} \varepsilon \varphi^{\prime}(t)+\frac{15}{12} \varepsilon^{2} \varphi^{\prime \prime}(t)+\mathcal{O}_{\infty}\left(\varepsilon^{3}\right) .
\end{aligned}
$$

Finally, we compute the shifted triple moving average for $k=0,1,2$. This yields

$$
\left(\begin{array}{c}
M_{\varphi}^{3}(t) \\
M_{\varphi}^{3}(t-\varepsilon) \\
M_{\varphi}^{3}(t-2 \varepsilon)
\end{array}\right)=\underbrace{\left(\begin{array}{rrr}
1 & -\frac{3}{2} & \frac{15}{12} \\
1 & -\frac{5}{2} & \frac{39}{12} \\
1 & -\frac{7}{2} & \frac{75}{12}
\end{array}\right)}_{D}\left(\begin{array}{c}
\varphi(t) \\
\varepsilon \varphi^{\prime}(t) \\
\varepsilon^{2} \varphi^{\prime \prime}(t)
\end{array}\right)+\mathcal{O}_{\infty}\left(\varepsilon^{3}\right)
$$



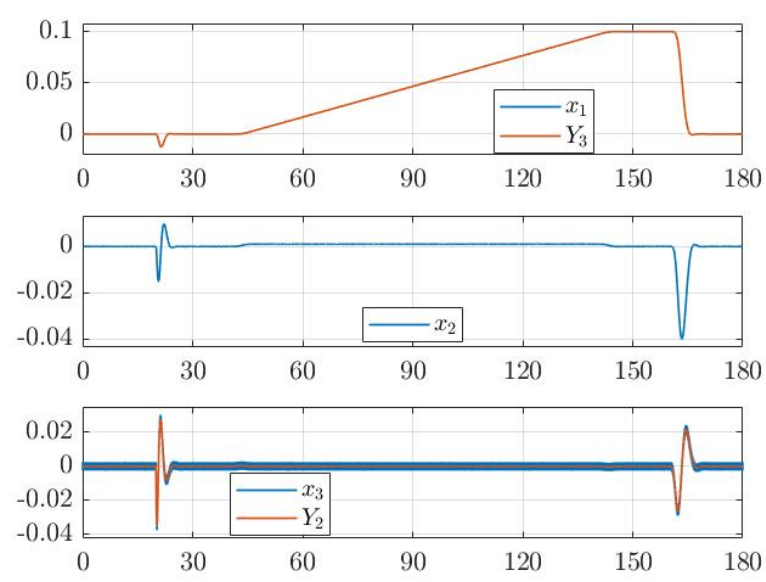

Fig. 1. $x_{1}$ and estimate $\hat{Y}_{3} / \varepsilon^{2}$ (top); $x_{2}$ (middle); $x_{3}$ and estimate $\hat{Y}_{2} / \varepsilon^{2}$ (bottom).
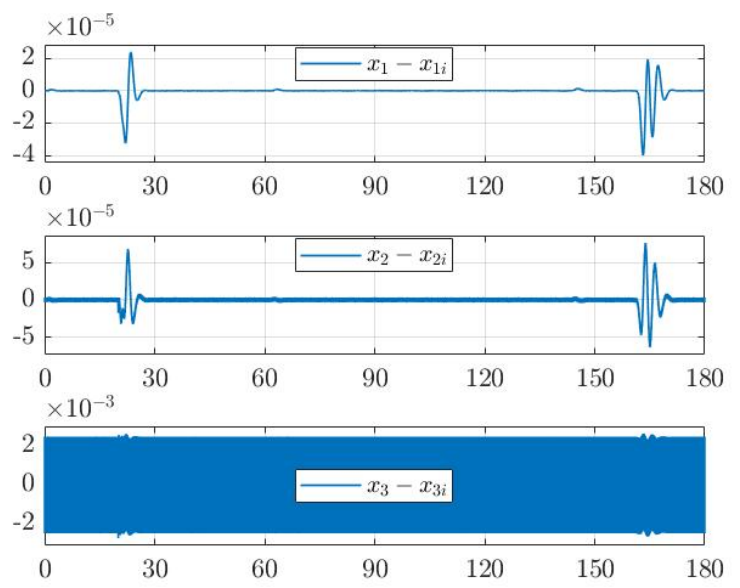

Fig. 2. Difference between $x$ (signal injection) and $x_{i}$ (ideal control).

Let $\alpha=\left(\begin{array}{lll}1 & 0 & 0\end{array}\right) D^{-1}=\left(\begin{array}{lll}17 / 4 & -5 & 7 / 4\end{array}\right)$. This yields the desired conclusion

$$
P_{\varphi}(t)=\alpha D \varphi(t)=\varphi(t)+\mathcal{O}_{\infty}\left(\varepsilon^{3}\right) .
$$

Combining the three lemmas, we estimate the $Y_{i}$.

Theorem 2: Considering the operator $P$ defined in lemma 3, we have an estimate for each of the $Y_{i}$, namely

$$
\begin{aligned}
\widehat{Y_{2}}(t) & :=\frac{1}{\overline{S_{1}^{2}}} P_{y \check{S}_{1}}(t) \\
& =Y_{2}(t)+\mathcal{O}_{\infty}\left(\varepsilon^{3}\right) \\
\widehat{Y_{3}}(t) & :=\frac{1}{\overline{s_{2}^{2}}} P_{y \check{s}_{2}}(t)-\frac{\left\langle s_{1}^{2}, s_{2}\right\rangle}{\overline{s_{2}^{2}}} \widehat{Y_{2}}(t) \\
& =Y_{3}(t)+\mathcal{O}_{\infty}\left(\varepsilon^{3}\right) \\
\widehat{Y_{1}}(t) & :=\frac{1}{\overline{s_{1}^{2}}} P_{y \check{s}_{1}}(t)-\frac{\left\langle s_{1}^{2}, s_{1}\right\rangle}{\overline{s_{1}^{2}}} \widehat{Y_{2}}(t) \\
& =Y_{1}(t)+\mathcal{O}_{\infty}\left(\varepsilon^{3}\right) \\
\widehat{Y_{0}}(t) & :=P_{y}(t)-\overline{s_{1}^{2}} \widehat{Y_{2}}(t) \\
& =Y_{0}(t)+\mathcal{O}_{\infty}\left(\varepsilon^{3}\right) .
\end{aligned}
$$
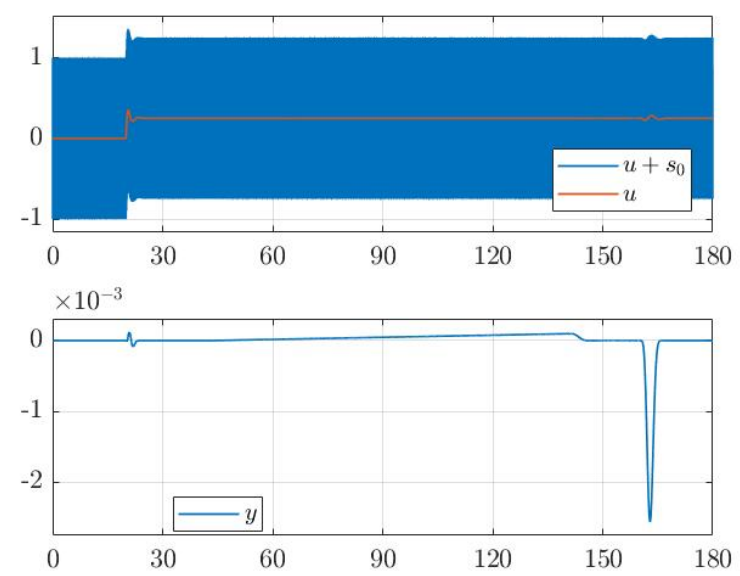

Fig. 3. Control (top) and measured output (bottom)

Proof: Let first determine the estimate for $Y_{2}$. Computing $P_{y \check{S}_{1}}$, we get

$$
\begin{aligned}
P_{y \check{S}_{1}}(t)= & P_{\widetilde{Y_{0}} \check{S}_{1}}(t)+P_{\widetilde{Y_{1} \check{S}_{1} \check{S}_{1}}}(t)+P_{\widetilde{Y}_{2}\left(\check{S}_{1}^{2}-\overline{S_{1}^{2}}\right)}(t) \\
& +P_{\widetilde{Y_{2}} \overline{S_{1}^{2}}}(t)+P_{\widetilde{Y_{3}} \check{s}_{2} \check{S}_{1}}(t)+\mathcal{O}_{\infty}\left(\varepsilon^{3}\right) .
\end{aligned}
$$

Since $P$ is a linear combination of shifted $M^{3}$ and $S_{1}, s_{1} S_{1}$, $s_{2} S_{1}$ and $S_{1}^{2}-\overline{S_{1}^{2}}$ have zero mean, we have by lemma 2

$$
P_{y \check{S}_{1}}(t)=P_{\widetilde{Y_{2}} \overline{S_{1}^{2}}}(t)+\mathcal{O}_{\infty}\left(\varepsilon^{3}\right) .
$$

Consequently, using lemma 3 , we have the following estimate for $\widetilde{Y_{2}}$ (which is equal to $Y_{2}$ )

$$
\widehat{Y_{2}}(t)=\frac{1}{\overline{S_{1}^{2}}} P_{y \check{S}_{1}}(t)=Y_{2}(t)+\mathcal{O}_{\infty}\left(\varepsilon^{3}\right) .
$$

Let compute $\widehat{Y_{3}}$. The same calculations for $P_{y \check{s}_{2}}$ provide

$$
P_{y \check{s}_{2}}(t)=\overline{s_{2}^{2} \widetilde{Y}_{3}}(t)=\overline{s_{2}^{2}}\left(Y_{3}(t)+\frac{\left\langle s_{1}^{2}, s_{2}\right\rangle}{\overline{s_{2}^{2}}} Y_{2}(t)\right),
$$

that is, by definition of $\widehat{Y_{3}}(14 b)$

$$
\widehat{Y_{3}}(t)=Y_{3}(t)+\mathcal{O}_{\infty}\left(\varepsilon^{3}\right) .
$$

Following the previous lines, we get the estimates $\widehat{Y_{0}}, \widehat{Y_{1}}$.

\section{A WORKED EXAMPLE}

We illustrate the interest of the method on the system

$$
\begin{aligned}
\dot{x}_{1} & =x_{2} \\
\dot{x}_{2} & =x_{3} \\
\dot{x}_{3} & =u+d \\
y & =x_{1} x_{2}+\frac{x_{3}^{3}}{3},
\end{aligned}
$$

where $d$ is an unknown disturbance. We would like $x_{1}$ to track the reference $x_{1}^{r e f}$ while rejecting the disturbance, with a time response of about a few time units. We want to operate around steady state, i.e. near states of the form $\left(x_{1}^{s s}, 0,0\right)$ and inputs such that $u^{s s}+d^{s s}=0$. Notice the system is not first-order observable around steady state because of the 

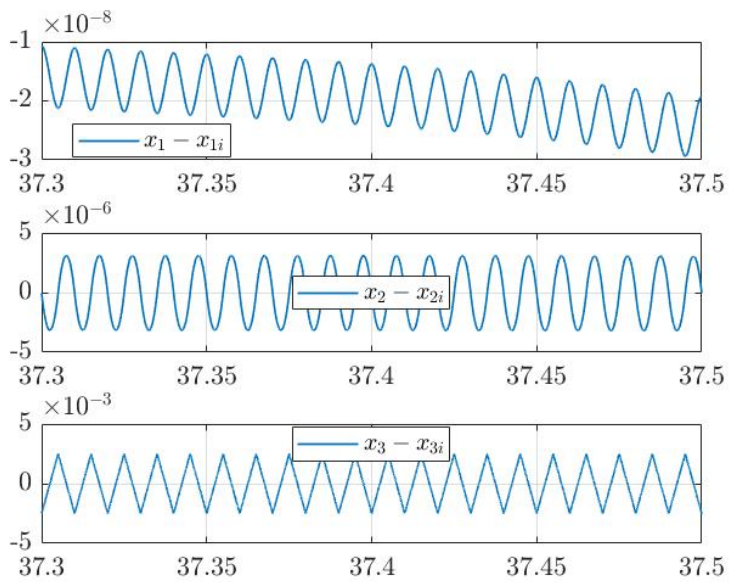

Fig. 4. Zoom on Fig. 2.
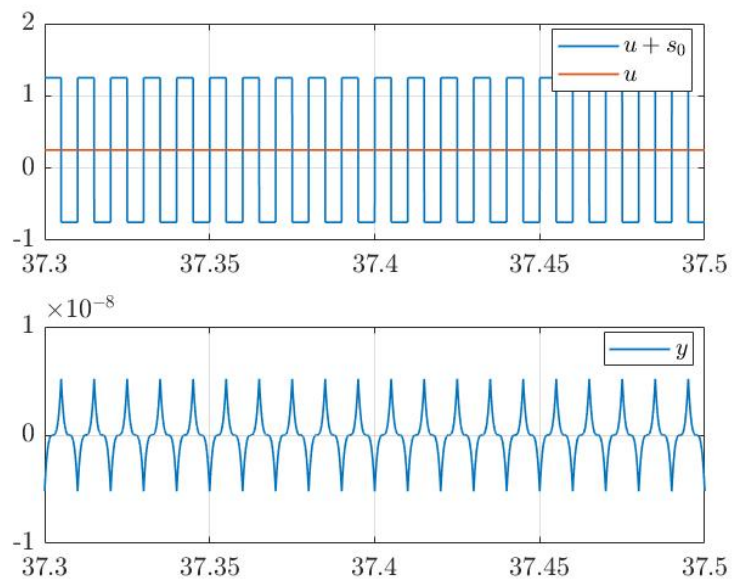

Fig. 5. Zoom on Fig. 3.

very degenerate output $y$, which makes the control problem far from obvious. Nonetheless, the virtual measurements are

$$
\begin{aligned}
Y_{1}=\varepsilon\left(\begin{array}{lll}
x_{2} & x_{1} & x_{3}^{2}
\end{array}\right)\left(\begin{array}{l}
0 \\
0 \\
1
\end{array}\right) & =\varepsilon x_{3}^{2} \\
Y_{2}=\frac{\varepsilon^{2}}{2}\left(\begin{array}{lll}
0 & 0 & 1
\end{array}\right)\left(\begin{array}{ccc}
0 & 1 & 0 \\
1 & 0 & 0 \\
0 & 0 & 2 x_{3}
\end{array}\right)\left(\begin{array}{l}
0 \\
0 \\
1
\end{array}\right) & =\varepsilon^{2} x_{3} \\
Y_{3}=\varepsilon^{2}\left(\begin{array}{lll}
x_{2} & x_{1} & x_{3}^{2}
\end{array}\right)\left(\begin{array}{l}
0 \\
1 \\
0
\end{array}\right) & =\varepsilon^{2} x_{1}
\end{aligned}
$$

hence first-order observability is restored thanks to $Y_{2}$ and $Y_{3}$, without even considering $y$ and $Y_{1}$. Notice thirdorder averaging is paramount, since the virtual measurement $Y_{1}$ stemming from second-order averaging is still degenerate.

With $Y_{3}=\varepsilon^{2} x_{1}$ and $Y_{2}=\varepsilon^{2} x_{3}$, the system is completely linear and can therefore be easily controlled. We design a
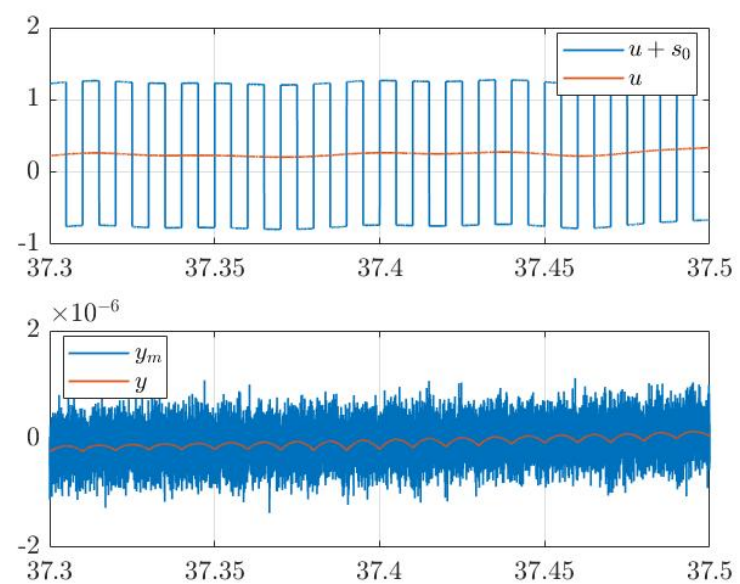

Fig. 6. Control (top) and measured output (bottom) with noise

classical controller-observer design, with observer

$$
\left(\begin{array}{c}
\dot{\hat{x}}_{1} \\
\dot{\hat{x}}_{2} \\
\dot{\hat{x}}_{3}
\end{array}\right)=\left(\begin{array}{c}
\hat{x}_{2} \\
\hat{x}_{3} \\
u
\end{array}\right)+L\left(\begin{array}{c}
Y_{3} / \varepsilon^{2}-\hat{x}_{1} \\
Y_{2} / \varepsilon^{2}-\hat{x}_{3}
\end{array}\right)
$$

and integral controller

$$
\begin{aligned}
u & =-k_{1} \hat{x}_{1}-k_{2} \hat{x}_{2}-k_{3} \hat{x}_{3}-k_{I} \eta_{I} \\
\dot{\eta}_{I} & =Y_{3}-x_{1}^{r e f} .
\end{aligned}
$$

The $3 \times 2$ matrix $L$ and $\left(k_{1}, k_{2}, k_{3}, k_{I}\right)$ are chosen such that the eigenvalues of the observer are $(-13.98,-1.57 \pm 1.59 i)$ and those of the controller are $(-1.20 \pm 1.77 i,-2.00 \pm 0.58 i)$, which ensures a time response of a few time units and a reasonable robustness; the observer is faster than the controller, in accordance with Loop Transfer Recovery (at the plant input). Setting $\eta:=\left(\hat{x}_{1}, \hat{x}_{2}, \hat{x}_{3}, \eta_{I}\right)^{T}$, this controllerobserver reads

$$
\begin{aligned}
& u=-K \eta \\
& \dot{\eta}=M \eta+N x_{1}^{r e f}(t)+L\left(\begin{array}{l}
Y_{3} / \varepsilon^{2} \\
Y_{2} / \varepsilon^{2}
\end{array}\right) .
\end{aligned}
$$

Following section II, this yields the implementable control law for (15a)-(15c), which is a particular case of (11),

$$
\begin{aligned}
& u=-K \eta+s_{0}\left(\frac{t}{\varepsilon}\right) \\
& \dot{\eta}=M \eta+N x_{1}^{r e f}(t)+L\left(\begin{array}{c}
\widehat{Y}_{3} / \varepsilon^{2} \\
\widehat{Y}_{2} / \varepsilon^{2}
\end{array}\right),
\end{aligned}
$$

where $\widehat{Y}_{2}$ and $\widehat{Y}_{3}$ are obtained from the actual measurement (15d) by the demodulation procedure of section III. The injected signal $s_{0}$ is a square wave of amplitude 1 and frequency 100 , which ensures the oscillation is fast with respect to the time constants of the closed-loop system; $n:=2$ is used in the demodulating filter.

The test scenario is the following: at $t=0$ the system starts at rest at the origin, with the reference $x_{1}^{\text {ref }}$ set to 0 ; at $t=20$, a step disturbance $d$ of magnitude -0.25 is applied; for $40 \leq t \leq 140, x_{1}^{r e f}$ is a slow (filtered) ramp with 

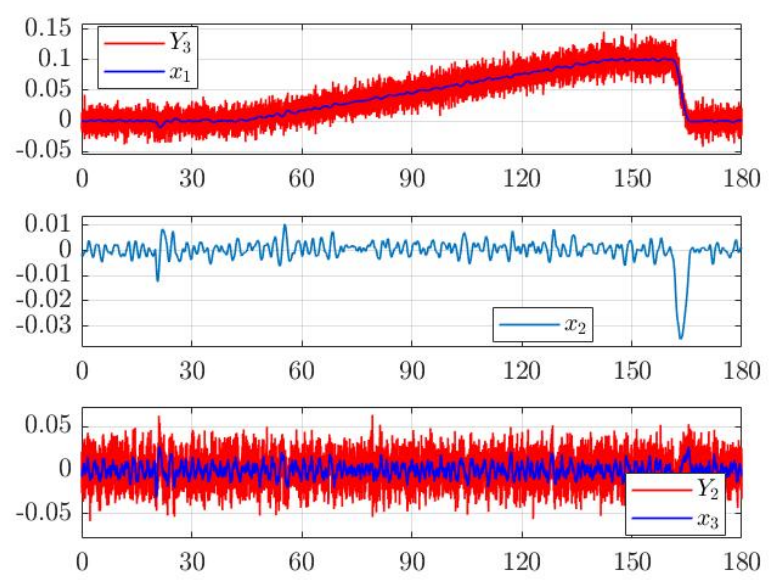

Fig. 7. $x_{1}$ and estimate $\hat{Y}_{3} / \varepsilon^{2}$ (top); $x_{2}$ (middle); $x_{3}$ and estimate $\hat{Y}_{2} / \varepsilon^{2}$ (bottom).

slope $10^{-3}$, meaning the system must slowly move while nearly first-order unobservable; finally, at $t=180, x_{1}^{r e f}$ is a filtered step so as to quickly return to 0 .

Without noise on the actual output $y$, the performance is excellent: the reference is tracked and the disturbance is rejected (Fig. 1). In fact the system behaves nearly as in the ideal situation where it is controlled directly from $x_{1}, x_{3}$ without signal injection (Fig. 2); the ripple is visible on $x_{3}$ because it is directly affected by the input, but is much smaller on $x_{1}, x_{2}$, as anticipated by the averaging analysis. A zoomed view for $37.3 \leq t \leq 37.5$ gives a better insight of the signals: Fig. 4 illustrates (6a); Fig. 5(top) shows the square wave in the control signal; Fig. 5(bottom) shows the ripple in the actual output is really tiny near steady state, with a strange form caused by the nonlinearity of this output.

With a measured output $y_{m}$ corrupted by noise, one thus might fear the ripple is much too small to be useful; this is not the case since the demodulation process is essentially a low-pass filter. Indeed, the system performs as desired (Fig. 7 and 8), even though the output ripple is buried into noise (Fig. 6). The measurement noise used in the simulation is a band-limited white noise with noise power $1 \times 10^{-18}$ and sample time $1 \times 10^{-5}$.

\section{CONCLusion}

We have shown that it is possible to produce "virtual outputs" thanks to the injection of fast-varying periodic
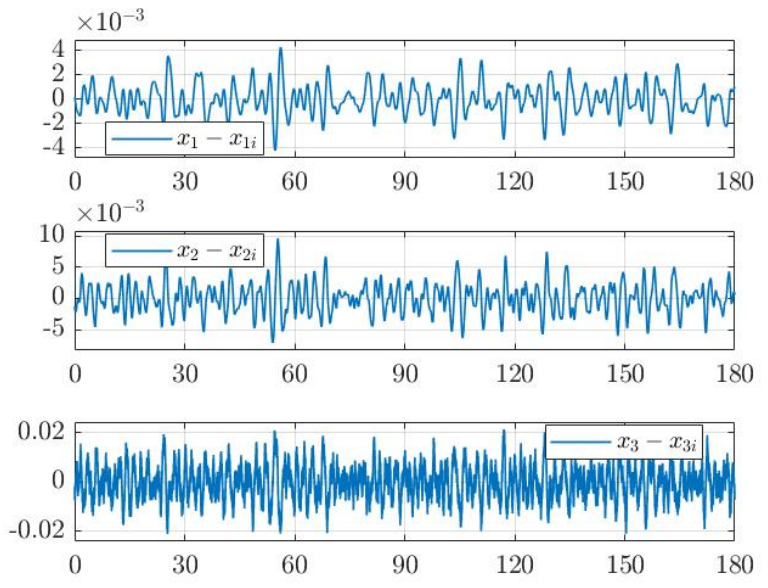

Fig. 8. Difference between $x$ (signal injection) and $x_{i}$ (ideal control).

probing signal. These virtual measurements can be used to simplify the design of a feeedback law, in particular when the system observability degenerates. The analysis relies on third-order averaging, and significantly extends the approach of [1]. For simplicity, we have restricted to Single-Input Single-Output systems with linear dynamics; in a future work we will generalize the approach to nonlinear Multi-Input Multi-Output systems.

\section{REFERENCES}

[1] P. Combes, A. K. Jebai, F. Malrait, P. Martin, and P. Rouchon, "Adding virtual measurements by signal injection," in American Control Conference, 2016, pp. 999-1005.

[2] P. Jansen and R. Lorenz, "Transducerless position and velocity estimation in induction and salient AC machines," IEEE Trans. Industry Applications, vol. 31, pp. 240-247, 1995.

[3] M. Corley and R. Lorenz, "Rotor position and velocity estimation for a salient-pole permanent magnet synchronous machine at standstill and high speeds," IEEE Trans. Industry Applications, vol. 34, pp. 784-789, 1998.

[4] A. K. Jebai, F. Malrait, P. Martin, and P. Rouchon, "Sensorless position estimation and control of permanent-magnet synchronous motors using a saturation model," International Journal of Control, vol. 89, no. 3, pp. 535-549, 2016.

[5] B. Yi, R. Ortega, and W. Zhang, "Relaxing the conditions for parameter estimation-based observers of nonlinear systems via signal injection," Systems and Control Letters, vol. 111, pp. 18-26, 2018.

[6] B. Yi, R. Ortega, H. Siguerdidjane, and W. Zhang, "An adaptive observer for sensorless control of the levitated ball using signal injection," in IEEE Conference on Decision and Control, 2019, pp. $6882-6887$.

[7] J. Sanders, F. Verhulst, and J. Murdock, Averaging methods in nonlinear dynamical systems, 2nd ed. Springer, 2005. 